\title{
Exacerbation risk and characterisation of the UK's asthma population from infants to old age
}

\author{
Chloe I Bloom, ${ }^{1}$ Francis Nissen, ${ }^{2}$ Ian J Douglas, ${ }^{2}$ Liam Smeeth, ${ }^{2}$ Paul Cullinan, ${ }^{1}$ \\ Jennifer K Quint ${ }^{1}$
}

- Additional material is published online only. To view please visit the journal online (http://dx.doi.org/10.1136/ thoraxjnl-2017-210650).

'Department of Respiratory Epidemiology, Occupational Medicine and Public Health, NHLI, Imperial College London, London, UK

${ }^{2}$ Department of Noncommunicable Disease Epidemiology, London School of Hygiene and Tropical Medicine, London, UK

\section{Correspondence to} Dr Chloe I Bloom, Department of Respiratory Epidemiology, Occupational Medicine and Public Health, NHLI, Imperial College London, London SW7 2AZ, UK; chloe.bloom06@ imperial.ac.uk

Received 15 June 2017 Revised 14 September 2017 Accepted 9 October 2017 Published Online First 26 October 2017

\begin{abstract}
Background Few studies have examined the characteristics of a general asthma population; most have focused on more severe patients or severe exacerbations.
\end{abstract}

Methods This population-based cohort study, from April 2007 to September 2015, used linked primary and secondary care electronic healthcare records (Clinical Practice Research Datalink, Hospital Episode Statistics). Characteristics of four age cohorts, 'Under $5 s^{\prime}$, ' 5 to $17 s$ ', '18 to 54s', '55+', were described. Exacerbation risk factors, including asthma severity (measured by the British Thoracic Society (BTS) stepwise approach), were assessed using Poisson regression.

Results 424326 patients with current asthma were eligible ( $n$, median follow-up: 'Under $5 s^{\prime}=17320,1$ year; ' 5 to $17 s^{\prime}=82707,3.3$ years; ' 18 to $54 s^{\prime}=210724,4$ years; ' $55+'=113575,5.1$ years). Over $60 \%$ of the total study population had mild asthma (BTS steps 1/2). There were differences between the cohort's characteristics, including by gender, disease severity and exacerbation pattern. The rate of exacerbations was highest in the oldest cohort and lowest in the ' 5 to $17 \mathrm{~s}$ ' cohort (rate per 10 person-years $(95 \% \mathrm{Cl})$, 'Under $5 s^{\prime}=4.27$ (4.18 to $4.38),{ }^{\prime} 5$ to $17 s^{\prime}=1.48$ (1.47 to 1.50$),{ }^{\prime} 18$ to $54 s^{\prime}=3.22$ (3.21 to 3.24$)$, ' $55+^{\prime}=9.40$ (9.37 to 9.42$)$ ). In all cohorts, exacerbation rates increased with increasing asthma severity, after adjusting for confounders including gender, socioeconomic status, smoking, body mass index, atopy, rhinitis, gastro-oesophageal reflux, anxiety, depression and COPD.

Conclusion The majority of UK patients with asthma had mild asthma and did not experience an exacerbation during follow-up. Patients aged $\geq 55$ years had the lowest proportion with mild asthma and highest rate of exacerbations; the opposite was found in patients aged between 5 and 18 years.

\section{INTRODUCTION}

Asthma is a common disease and its lifetime prevalence in the UK continues to rise. ${ }^{1}$ Internationally, the UK ranks as having one of the highest prevalence and mortality rates in Europe. ${ }^{2}{ }^{3}$ Asthma causes a significant burden to the National Health Service (NHS) across the spectrum of ages, with 5.4 million people receiving treatment and approximately 65000 hospital admissions yearly. ${ }^{4}$ In addition, it leads to a significant societal burden including absence from work and school.

Most patients with asthma do not require secondary care intervention, yet there is limited

\section{Key messages}

What is the key question?

- What are the characteristics of the UK's general asthma population, including all phenotypes and ages, and how are these associated with the risk of an exacerbation?

What is the bottom line?

- Many differences in demographics, clinical characteristics and exacerbation rates were found between the four generations of patients with asthma; the oldest ( $\geq 55$ years) cohort, followed by the youngest ( $<5$ years) cohort, had the most severe asthma with the highest exacerbation rates.

Why read on?

- This is the first description of the UK's general asthma population and includes all those with current asthma, from infants to old age.

knowledge on the characterisation of national asthma populations. To date, epidemiological studies have focused on patients with more severe disease, distinct phenotypes, or have separated those with childhood-onset from adult-onset asthma. In addition, most published reports on exacerbations have analysed only hospital admissions or Accident \& Emergency (A\&E) visits. Currently, we do not have comprehensive knowledge on the UK's general asthma population; this is needed to help us understand the natural history of asthma and guide population-level public health measures.

Over $98 \%$ of the UK population is registered with a general practitioner (GP). ${ }^{5}$ Most patients with asthma first present to their GP who makes the diagnosis based on their respiratory symptoms, signs and test results; in patients with insufficient evidence, such as children under 5 years who cannot perform spirometry, a period of watchful waiting or monitored treatment may be advocated. Primary care electronic healthcare records, originating from routine clinical practice, capture this and a huge amount of other clinical and demographic information in a longitudinal record.

We have used national electronic healthcare records to describe, for the first time, the UK's asthma population, laying out the differences and similarities that exist between the generations using four consecutive age cohorts between infancy and 


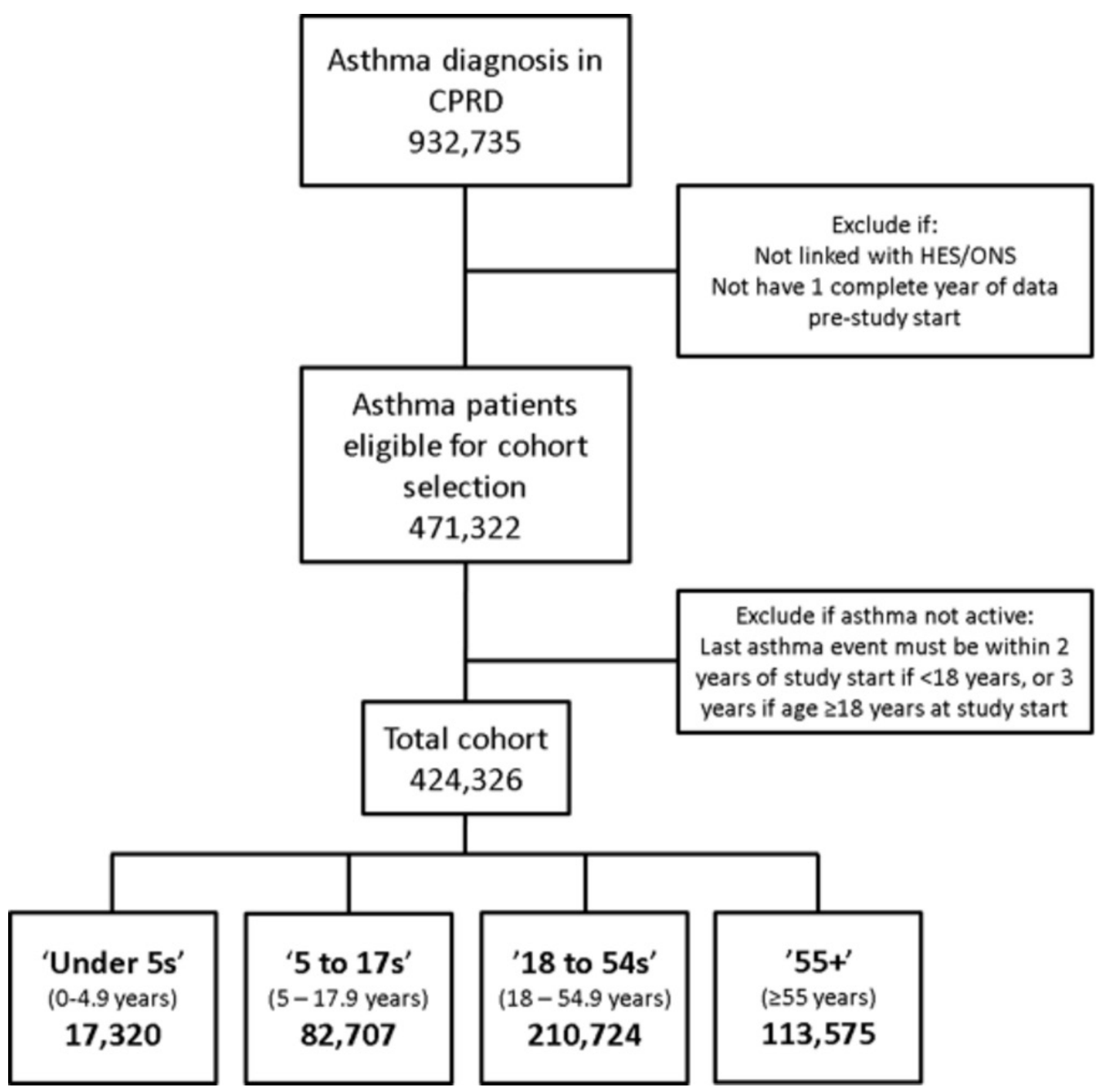

Figure 1 Flow diagram showing eligibility criteria and patient numbers. CPRD, Clinical Practice Research Datalink; HES, Hospital Episode Statistics; ONS, Office of National Statistics.

old age. We have assessed several factors that may be associated with an increased risk of an exacerbation.

\section{METHODS}

\section{Data sources}

Clinical Practice Research Datalink (CPRD) included 674 GP practices and current coverage of over 11.3 million patients who represent the UK's population with respect to age, gender, body mass index (BMI) and ethnicity. ${ }^{6}$ Approximately $60 \%$ of CPRD practices have patient-level linkage to Hospital Episode Statistics data (collected during a patient's visit to an NHS hospital), Index of Multiple Deprivation (IMD) and Office of National Statistics mortality data.

\section{Study design and population}

We conducted a population-wide open cohort study. The study population consisted of patients in CPRD-linked practices with validated Read codes (a clinical terminology system) indicating prevalent or incident asthma. ${ }^{7}$ Patients were eligible if a code for asthma had been recorded at $\leq 2$ years prior to study entry if aged $<18$ years, or $\leq 3$ years if aged $\geq 18$ years (figure 1 ). Patients entered the study at the latest of their asthma diagnosis date, the date the practice began recording research quality data, or 1 April 2007. Patient follow-up was censored at the earliest of 30 September 2015, death, transfer out of CPRD practice, linkage end date or practice last collection date. The patient's age at study entry dictated which cohort they entered. Age categories were based on the age distinctions used in the British Thoracic Society (BTS) asthma guidance ${ }^{8}$ : under 5 years old, 5-12 years old and adolescents were combined in the 5-17s year cohort, and the adult cohort was split into two cohorts due to the possible confounding from COPD after 55 years old.

\section{Outcome and variables}

The main outcome was asthma exacerbations. An exacerbation was defined as $\leq 300 \mathrm{mg}$ oral corticosteroids (OCS) (not prescribed during an annual asthma review), or an A\&E visit, or hospital admission. A cut-off of $\leq 150 \mathrm{mg}$ OCS was used for children $<5$ years old. Exacerbations recorded within 14 days after the index 1 were considered part of the same exacerbation. Level of care of each exacerbation was documented as the highest level per episode. Incident lower respiratory tract infections treated with antibiotics (Ax-LRTI) were also measured by identifying specific Read codes (available upon request) with same-day prescription of appropriate antibiotics.

BMI was measured using $\mathrm{kg} / \mathrm{m}^{2}$ (z-scores were used if $<5$ years old). A history of atopy, rhinitis, gastro-oesophageal reflux (reflux), anxiety and depression was recorded using appropriate Read codes (available upon request). COPD was classified by Read codes, a smoking history and age $>35$ years. ${ }^{9}$ The 
BTS stepwise approach (incorporating inhaler class and dose) is a recommended evidence-based method of measuring asthma severity. ${ }^{8}$ Using the 2016 guidelines, patients were classified by their highest BTS step (most severe asthma) using all medications prescribed during the year before their study start date. Step 6 is 'continuous or frequent' OCS use; we defined this as $\geq 6$ annual OCS prescriptions that must have occurred in $\geq 2$ yearly quarters (a yearly quarter was 3 consecutive months). Approximately $20 \%$ of inhaled corticosteroid (ICS) prescriptions did not have a precise dose recorded; using the BTS guidelines and the type of ICS device and dosage prescribed, the ICS dose levels were imputed with good accuracy.

\section{Statistical analysis}

Baseline characteristics were tabulated for each cohort. To take into account multiple exacerbations, a repeated measures Poisson regression model was used to calculate crude rate ratios of exacerbations. This model was also used to determine the relative rates of exacerbations by BTS step, adjusting for: gender, age, IMD (socioeconomic index, 1 is least deprived), BMI, smoking status, atopy, rhinitis, reflux, anxiety, depression and COPD status. Where $>20 \%$ of the data were missing the variable was excluded from the regression model. The data were also modelled using Poisson regression and time to first exacerbation during study follow-up; results were displayed by Kaplan-Meier graphs.

\section{RESULTS}

\section{Patient characteristics}

In the total study population there were 424326 patients with current asthma. The number of patients and follow-up varied between cohorts; the smallest and shortest follow-up was in the 'Under 5s' and the largest, with a longer follow-up, was the ' 18 to $54 \mathrm{~s}$ ' cohort ('Under $5 \mathrm{~s}$ ' $\mathrm{n}=17320$, IQR 0.5-1.8 years; '5 to $17 \mathrm{~s}$ ' $\mathrm{n}=82707$, IQR $1.4-5.8$; ' 18 to $54 \mathrm{~s}$ ' $\mathrm{n}=210724$, IQR 1.7-6.9 years; ' $55+$ ' $\mathrm{n}=113575$, IQR 2.4-7.6) (table 1). Female prevalence increased with increasing cohort age and became the dominant gender from 18 years onwards (table 1). Over 70\% in the 'Under 5s' cohort were aged between 3 and 5 years (table 1 ). In the ' $55+$ ' cohort, the numbers of patients were inversely proportional to the age group. The highest percentage of smokers was in the ' 18 to 54 's' cohort (current $25.9 \%$ ) and ex-smokers in the ' $55+$ ' cohort (ex-smoker 49.2\%) (table 1). Recorded BMI was predominantly either normal or underweight if cohort age was $<18$ years, but normal or above if cohort age was $\geq 18$ years (table 1 ).

Taking the total cohort as a whole (all ages), 35\% (149 338) of patients were not taking regular asthma medication and $27 \%$ (112 937) were taking an ICS at the lowest dose appropriate for their age. The proportion in each BTS step varied with each cohort (table 1 and figure 2). Around 70\% of patients $<55$ years old were in BTS step 1 or 2, only in the 'Under $5 \mathrm{~s}$ ' cohort were there more in BTS step 2 than 1 (table 1). The ' $55+$ ' cohort had the smallest proportion with patients on BTS step 1 or $2(44 \%)$. All the cohorts had a very low proportion in BTS step $6(<2 \%)$.

The proportion of patients with atopy was highest in the two youngest cohorts ( $<18$ years) (table 1$)$. The proportion of patients with rhinitis, reflux, anxiety or depression was highest in the two oldest cohorts ( $\geq 18$ years) (table 2 ). Just under one quarter aged $\geq 55$ years had a coexistent diagnosis of COPD (table 1); of these, $66 \%$ had a COPD diagnosis after their asthma diagnosis.

\section{Exacerbation characteristics}

The rate of exacerbations was highest in the oldest age group, followed by the youngest age group, and lowest in the ' 5 to $17 \mathrm{~s}$ ' cohort (exacerbations per 10 person-years: ' $55+$ ' $=9.4(95 \% \mathrm{CI}$ 9.37 to 9.42 ), 'Under $5 \mathrm{~s}$ ' $=4.27$ (95\% CI 4.18 to 4.38 ), ' 18 to $54 \mathrm{~s}$ ' $=3.22(95 \%$ CI 3.21 to 3.24$)$, ' 5 to $17 \mathrm{~s}$ ' $=1.48$ (95\% CI 1.47 to 1.5$)$ ) (table 2 ). Of those who did exacerbate, the majority had $\leq 1$ exacerbation per year, in cohorts aged $\geq 5$ years (annual frequency $\leq 1 /$ year: ' 5 to $17 \mathrm{~s}$ ' $=86.9 \%$; ' 18 to $54 \mathrm{~s}$ ' $=81.3 \%$; ' $55+$ ' $=65 \%)$; the opposite was found in the 'Under $5 \mathrm{~s}$ ' cohort, in whom just over half experienced $>1$ exacerbation per year $(54.7 \%$ ) (table 2 and figure 3). Around $85 \%$ of exacerbations in each cohort did not require overnight hospitalisation ('Under $5 \mathrm{~s}$ ' $=85.6 \%$, ' 5 to $17 \mathrm{~s}$ ' $=85.2 \%$, ' 18 to $54 \mathrm{~s}$ ' $=83.1 \%$, ' $55+$ ' $=88.7 \%)$. All patients were most likely to see their GP as their maximum level of care; the oldest age cohort had a lower proportion of hospitalised exacerbations, but highest proportion of asthma deaths (table 2).

The rate of Ax-LRTIs was much lower than the rate of exacerbations; the rate was highest in the ' $55+$ ' cohort and lowest in the ' 5 to $17 \mathrm{~s}$ ' cohort (' $55+$ ' $=1.93$ (95\% CI 1.92 to 1.94 ) exacerbations per 10 person-years, ' 5 to $17 \mathrm{~s}$ ' $=0.22(95 \% \mathrm{CI}$ 0.21 to 0.22 ) exacerbations per 10 person-years). Of those with an Ax-LRTI, $<10 \%$ occurred at the time of, or 14 days after, an exacerbation ('Under $5 \mathrm{~s}$ ' $=5 \%$, ' 5 to $17 \mathrm{~s}$ ' $=4.9 \%$, ' 18 to $54 \mathrm{~s}$ ' $=8.3 \%, ' 55+{ }^{\prime}=7.3 \%$ ) and $\leq 1 \%$ of Ax-LRTIs preceded an exacerbation within 14 days ('Under $5 \mathrm{~s}$ ' $=0.6 \%$, '5 to $17 \mathrm{~s}$ ' $=0.4 \%$, ' 18 to $54 \mathrm{~s}^{\prime}=0.9 \%,{ }^{\prime} 55+{ }^{\prime}=1 \%$ ).

The rate of exacerbations increased with increasing asthma severity (BTS step) in every cohort, with the lowest rate, 0.73 (95\% CI 0.7 to 0.77 ) exacerbations per 10 person-years, in BTS step 1 in ' 5 to 17 ' cohort and the highest rate, 60.2 (95\% CI 57.97 to 62.52 ) exacerbations per 10 person-years, in BTS step 6 in the ' $55+$ ' cohort (table 3 ).

\section{Effect of demographic and clinical characteristics on exacerbation rates (univariable analysis)}

Female gender was significantly associated with exacerbation risk in all cohorts except for the ' 5 to $17 \mathrm{~s}$ ' cohort; the relative rate decreased in the 'Under $5 \mathrm{~s}$ ' but increased in the oldest two cohorts (Female: 'Under 5s' incidence rate ratio $($ IRR $)=0.84$ (95\% CI 0.76 to 0.92 ), ' 18 to 54 s' IRR $=1.67$ (95\% CI 1.64 to 1.71 ), ' 55 +' IRR $=1.11$ (95\% CI 1.09 to 1.13 ), $\mathrm{p}<0.001$ ). Within the oldest two cohorts, as age increased, exacerbation rates also increased; the opposite occurred in the youngest two cohorts (see online supplementary table S1-4). Current and ex-smoking was significantly associated with an increased rate in patients $\geq 18$ years old (online supplementary table S1-4). Patients with a 'non-normal' BMI (underweight, overweight or obese) had higher exacerbation rates in the ' 5 to $17 \mathrm{~s}$ ' and '18 to 54s' cohorts (online supplementary table S1-4). Having atopy, rhinitis, reflux, anxiety or depression increased the relative rate of exacerbations in all cohorts (online supplementary table S1-4). Exacerbation rates were also higher in patients with asthma with COPD (online supplementary tables S3 and S4).

\section{Effect of asthma severity on exacerbation rates (multivariable analysis modelling on all exacerbations)}

Each increase in BTS step was significantly associated with an increased rate of exacerbations compared with BTS step 1 (figure 4). This effect was seen in all cohorts, but the largest adjusted rate ratios were found in the youngest cohort, and the smallest were found in the oldest cohort ('Under 5s' cohort: step 
Table 1 Description of each cohort

\begin{tabular}{|c|c|c|c|c|c|c|c|c|}
\hline & \multicolumn{2}{|l|}{ Under $5 \mathrm{~s}$} & \multicolumn{2}{|l|}{5 to $17 \mathrm{~s}$} & \multicolumn{2}{|l|}{18 to $54 \mathrm{~s}$} & \multicolumn{2}{|l|}{$55+$} \\
\hline & $\mathrm{N}$ & $\%$ & $\mathrm{~N}$ & $\%$ & $\mathrm{~N}$ & $\%$ & $\mathrm{~N}$ & $\%$ \\
\hline Total & 17320 & & 82707 & & 210724 & & 113575 & \\
\hline F/U median (IQR, years) & $1(0.5-1.8)$ & & $3.3(1.4-5.8)$ & & $4.0(1.7-6.9)$ & & $5.1(2.4-7.6)$ & \\
\hline \multicolumn{9}{|l|}{ Gender } \\
\hline Female & 6635 & 38.3 & 35993 & 43.5 & 120910 & 57.4 & 67865 & 59.8 \\
\hline \multicolumn{9}{|l|}{ Age category* } \\
\hline 1 & 943 & 5.4 & 13687 & 16.6 & 37322 & 17.7 & 46232 & 40.7 \\
\hline 2 & 3738 & 21.6 & 19370 & 23.4 & 59239 & 28.1 & 37226 & 32.8 \\
\hline 3 & 5606 & 32.4 & 27619 & 33.4 & 62750 & 29.8 & 23460 & 20.7 \\
\hline 4 & 7033 & 40.6 & 22031 & 26.6 & 51413 & 24.4 & 6657 & 5.9 \\
\hline \multicolumn{9}{|l|}{ IMDt } \\
\hline 1 & 3472 & 20.1 & 17987 & 21.8 & 45058 & 21.4 & 24310 & 21.4 \\
\hline 2 & 3367 & 19.4 & 17044 & 20.6 & 45241 & 21.5 & 27151 & 23.9 \\
\hline 3 & 3102 & 17.9 & 15585 & 18.8 & 41377 & 19.6 & 23458 & 20.7 \\
\hline 4 & 3772 & 21.8 & 16765 & 20.3 & 42673 & 20.3 & 21356 & 18.8 \\
\hline 5 & 3552 & 20.5 & 15283 & 18.5 & 36212 & 17.2 & 17220 & 15.2 \\
\hline Missing & 55 & 0.3 & 43 & 0.1 & 163 & 0.1 & 80 & 0.1 \\
\hline \multicolumn{9}{|l|}{ Smoking } \\
\hline Never & NA & NA & 61506 & 74.4 & 102684 & 48.7 & 42815 & 37.7 \\
\hline Current & NA & NA & 3476 & 4.2 & 54669 & 25.9 & 14826 & 13.1 \\
\hline Ex & NA & NA & 1151 & 1.4 & 53052 & 25.2 & 55903 & 49.2 \\
\hline Missing & NA & NA & 16574 & 20.0 & 319 & 0.2 & 31 & 0.0 \\
\hline \multicolumn{9}{|l|}{ BMI } \\
\hline Normal & 7980 & 46.1 & 23068 & 27.9 & 69020 & 32.8 & 29495 & 26.0 \\
\hline Overweight & 1018 & 5.9 & 6393 & 7.7 & 58786 & 27.9 & 39274 & 34.6 \\
\hline Obese & 638 & 3.7 & 4105 & 5.0 & 54702 & 26.0 & 36189 & 31.9 \\
\hline Underweight & 683 & 3.9 & 24589 & 29.7 & 3879 & 1.8 & 2212 & 2.0 \\
\hline Missing & 7001 & 40.4 & 24552 & 29.7 & 24337 & 11.6 & 6405 & 5.6 \\
\hline \multicolumn{9}{|l|}{ BTS steps } \\
\hline 1 & 4889 & 28.2 & 33109 & 40.0 & 86360 & 41.0 & 24980 & 22.0 \\
\hline 2 & 6856 & 39.6 & 26766 & 32.4 & 54773 & 26.0 & 24542 & 21.6 \\
\hline 3 & 979 & 5.7 & 3785 & 4.6 & 18661 & 8.9 & 12321 & 10.9 \\
\hline 4 & 2227 & 12.9 & 11782 & 14.3 & 32821 & 15.6 & 25714 & 22.6 \\
\hline 5 & 97 & 0.6 & 1148 & 1.4 & 14663 & 7.0 & 19425 & 17.1 \\
\hline 6 & $<5$ & 0.0 & 22 & 0.0 & 623 & 0.3 & 1629 & 1.4 \\
\hline Non-BTS & 480 & 2.8 & 2020 & 2.4 & 2547 & 1.2 & 4613 & 4.1 \\
\hline Missing & 1790 & 10.3 & 4075 & 4.9 & 276 & 0.1 & 351 & 0.3 \\
\hline \multicolumn{9}{|l|}{ Atopy } \\
\hline Yes & 4883 & 28.2 & 25714 & 31.1 & 54205 & 25.7 & 25629 & 22.6 \\
\hline \multicolumn{9}{|l|}{ Rhinitis } \\
\hline Yes & 629 & 3.6 & 6948 & 8.4 & 23740 & 11.3 & 14366 & 12.7 \\
\hline \multicolumn{9}{|l|}{ GORD } \\
\hline Yes & 764 & 4.4 & 1854 & 2.2 & 17150 & 8.1 & 19755 & 17.4 \\
\hline \multicolumn{9}{|l|}{ Anxiety } \\
\hline Yes & 290 & 1.7 & 4260 & 5.2 & 32844 & 15.6 & 19610 & 17.3 \\
\hline \multicolumn{9}{|l|}{ Depression } \\
\hline Yes & NA & NA & 2238 & 2.7 & 45489 & 21.6 & 23397 & 20.6 \\
\hline \multicolumn{9}{|l|}{ COPD } \\
\hline Yes & NA & NA & NA & NA & 5756 & 2.7 & 25781 & 22.7 \\
\hline
\end{tabular}

*Age categories are for 'Under 5s': 1 (1-1.9 years), 2 (2-2.9 years), 3 (3-3.9 years), 4 (4-4.9 years); '5 to 17s': 1 (5-7 years), 2 (8-10 years), 3 (11-13 years), 4 (14-17 years); '18 to 54s': 1 (18-24 years), 2 (25-34 years), 3 (35-44 years), 4 ( $45-54$ years); ' $55++^{\prime}: 1$ ( $55-64$ years), 2 (65-74 years), 3 (75-84 years), 4 ( $\geq 85$ years).

tSocioeconomic deprivation scale, 1 is least deprived. BTS steps are defined as $1=$ no regular preventer, $2=$ lowest ICS dose appropriate for age (or LTRA alone if $<5$ years), $3=$ add LABA (add LTRA if $<5$ years), $4=$ increase ICS dose to next level (medium in adults, low dose in children), may add in other therapy (adults: LTRA, theophylline, LAMA; children: LTRA), $5=$ =increase ICS dose (high in adults, medium in children), add fourth drug (adults: LTRA, theophylline, beta agonist tablet, LAMA; children: theophylline), $6=$ same ICS dose and continuous or frequent use of oral steroids. Children are patients aged below 12 years.

LAMA; children: theophylline), $6=$ same ICS dose and continuous or frequent use of oral steroids. Children are patients aged below 12 years.
BMI, body mass index; BTS, British Thoracic Society; F/U, follow-up; GORD, gastro-oesophageal reflux disease; ICS, inhaled corticosteroid; LABA, long acting beta-2 agonist; LAMA, long acting muscarinic antagonist; LTRA, leukotrienereceptor antagonist; NA, not applicable

$2 \mathrm{IRR}=2.3(95 \%$ CI 2.0 to 2.6$)$, step $3 \mathrm{IRR}=4.5(95 \%$ CI 3.7 to 5.5), step $4 \mathrm{IRR}=4(95 \%$ CI 3.5 to 4.7$)$, step $5 \mathrm{IRR}=4.7(95 \%$ CI 2.7 to 8.1 ), step 6 IRR $=16.3$ (95\% CI 0.8 to 348 ), adjusted for age, gender, IMD, atopy, rhinitis, reflux and anxiety; ' $55+$ ' cohort: step 2 IRR $=1.3(95 \%$ CI 1.2 to 1.3$)$, step 3 IRR $=1.6$ (95\% CI 1.5 to 1.6$)$, step $4 \mathrm{IRR}=2(95 \%$ CI 1.9 to 2$)$, step 


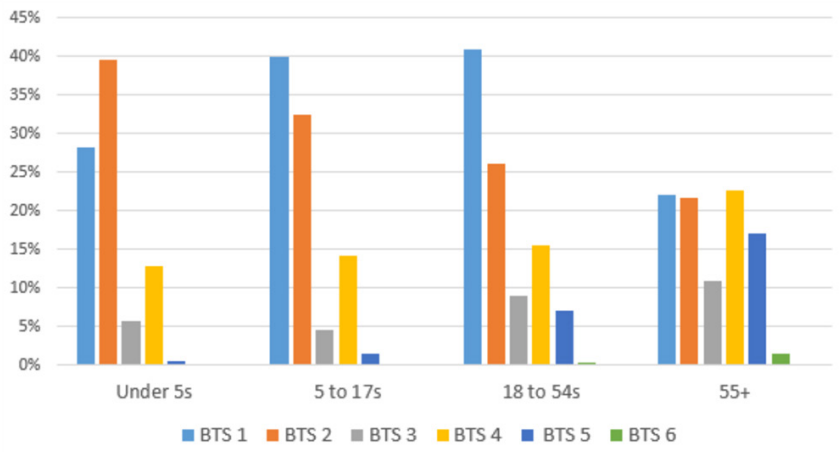

Figure 2 British Thoracic Society (BTS) steps by age cohort.

$5 \mathrm{IRR}=2.3(95 \%$ CI 2.2 to 2.3$)$, step $6 \mathrm{IRR}=10(95 \%$ CI 9.3 to 10.7), adjusted for age, gender, IMD, BMI, atopy, rhinitis, reflux, anxiety, depression and COPD) (figure 4).

\section{Effect of asthma severity on time to first exacerbation during study follow-up}

Time to first exacerbation analysis revealed a comparable pattern to analyses including all exacerbations. The Kaplan-Meier curves were steepest, with the shortest median times to first exacerbation, with each increasing BTS step (figure 5; online supplementary tables S2). In general, the curves were also steepest in the following cohort order: ' $55+$ ', 'Under $5 \mathrm{~s}$ ', ' 18 to $54 \mathrm{~s}$ ' and '5 to 17s' (figure 5; online supplementary table S2).

\section{DISCUSSION}

We have undertaken the first descriptive study of the UK's general asthma population and found many similarities and differences between the characteristics of cohorts representing: infants to preschoolers (Under $5 \mathrm{~s}$ ), young children to teenagers ( 5 to $17 \mathrm{~s}$ ), younger adults to middle age (18 to $54 \mathrm{~s}$ ) and middle

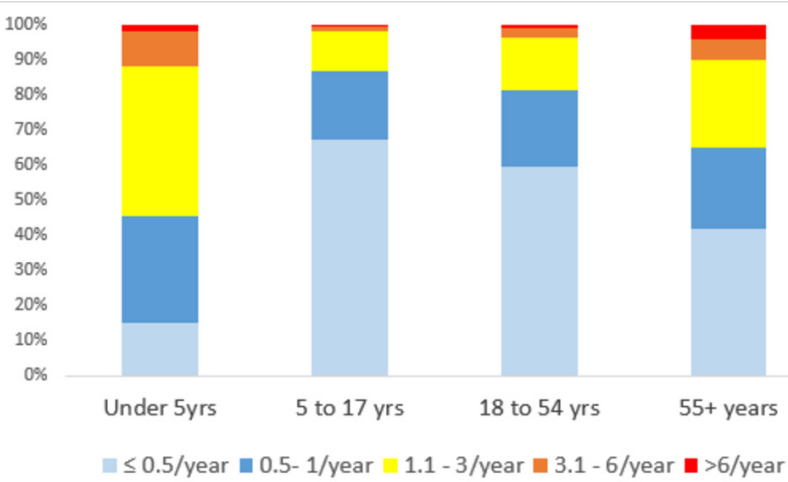

Figure 3 Bar chart of the patients who exacerbated during follow-up according to their annual frequency of exacerbations.

to old age $(55+)$. One clear difference was gender; maleswere found to be more prevalent in the younger cohorts, $<18$ years, but the opposite was found in the older cohorts; these results are in agreement with other prevalence, as well as incidence, asthma studies. ${ }^{1011}$ Smoking prevalence increased between the ' 5 to $17 \mathrm{~s}$ ' and ' 18 to $54 \mathrm{~s}$ ' cohorts but fell in the ' $55+$ ' cohort, in keeping with UK smoking data. ${ }^{12}$ Adult BMI ( $\geq 18$ years) increased with increasing cohort age, compatible with published national data. ${ }^{13}$ Atopy prevalence was highest in the two youngest cohorts $(<18$ years), which appears consistent with studies showing that lateonset asthma is less likely to be allergic asthma. ${ }^{14}$ A history of rhinitis and reflux both showed a steady increase with age; presently, there is a paucity of published data on the relationship between age and these disorders.

The majority of patients with asthma $(>60 \%$ of the total study population) had mild asthma, BTS step 1 or 2 ; it is notable that this cohort only included patients with current asthma and excluded patients who had not visited their GP practice for their

Table 2 Summary of exacerbations and presumed bacterial LRTIs for each cohort

\begin{tabular}{|c|c|c|c|c|c|c|c|c|}
\hline & \multicolumn{2}{|l|}{ Under 5s } & \multicolumn{2}{|l|}{5 to $17 \mathrm{~s}$} & \multicolumn{2}{|l|}{18 to $54 \mathrm{~s}$} & \multicolumn{2}{|l|}{$55+$} \\
\hline & Patients (\%) & $\begin{array}{l}\text { Rate per } \\
10 \text { person- } \\
\text { years }(95 \% \mathrm{Cl})\end{array}$ & Patients (\%) & $\begin{array}{l}\text { Rate per } \\
10 \text { person-years } \\
(95 \% \mathrm{Cl})\end{array}$ & Patients (\%) & $\begin{array}{l}\text { Rate per } \\
10 \text { person-years } \\
(95 \% \mathrm{Cl})\end{array}$ & Patients (\%) & $\begin{array}{l}\text { Rate per } \\
10 \text { person-years } \\
(95 \% \mathrm{Cl})\end{array}$ \\
\hline Total cohort & 17320 & & 82707 & & 210724 & & 113575 & \\
\hline Exacerbations & 7574 & 4.27 (4.18 to 4.38$)$ & 39970 & $1.48(1.47$ to 1.50$)$ & 245077 & $3.22(3.21$ to 3.24$)$ & 435029 & 9.40 (9.37 to 9.42$)$ \\
\hline Patients who exacerbated & $3706\left(21.4^{*}\right)$ & & $18449\left(22.3^{*}\right)$ & & $80041\left(38^{*}\right)$ & & $70954\left(62.5^{*}\right)$ & \\
\hline \multicolumn{9}{|c|}{ Annual exacerbation frequency } \\
\hline$\leq 0.5$ & 553 & & 12433 & & 47588 & & 29620 & \\
\hline $0.5-1$ & 1131 & & 3600 & & 17423 & & 16439 & \\
\hline $1.1-3$ & 1577 & & 2047 & & 12156 & & 17569 & \\
\hline $3.1-6$ & 369 & & 292 & & 2121 & & 4419 & \\
\hline$>6$ & 76 & & 77 & & 753 & & 2907 & \\
\hline \multicolumn{9}{|l|}{ Level of care } \\
\hline GP only & 5211 & & 26543 & & 155054 & & 334850 & \\
\hline$A \& E$ & 232 & & 984 & & 2257 & & 561 & \\
\hline Hospitalisation ( $<1$ day) & 966 & & 6515 & & 46227 & & 50385 & \\
\hline Hospitalisation ( $\geq 1$ day) & 1165 & & 5925 & & 41502 & & 49015 & \\
\hline Died & $<5$ & & $<5$ & & 37 & & 218 & \\
\hline Antibiotic-treated LRTIs & 2179 & 0.61 (0.58 to 0.64$)$ & 11488 & 0.22 (0.21 to 0.22$)$ & 81084 & 0.71 (0.71 to 0.72 ) & 171352 & $1.93(1.92$ to 1.94$)$ \\
\hline Patients with Ax-LRTI & $1612\left(9.3^{*}\right)$ & & $8252\left(10^{*}\right)$ & & $41874\left(19.9^{*}\right)$ & & $34656\left(30.5^{*}\right)$ & \\
\hline
\end{tabular}

*Per cent of total cohort, ; annual frequency is the number of patients who exacerbated within each band of annual frequency.

$A \& E$, Accident \& Emergency; Ax-LRTI, antibiotic-treated lower respiratory tract infection; GP, general practitioner; LRTI, lower respiratory tract infection. 


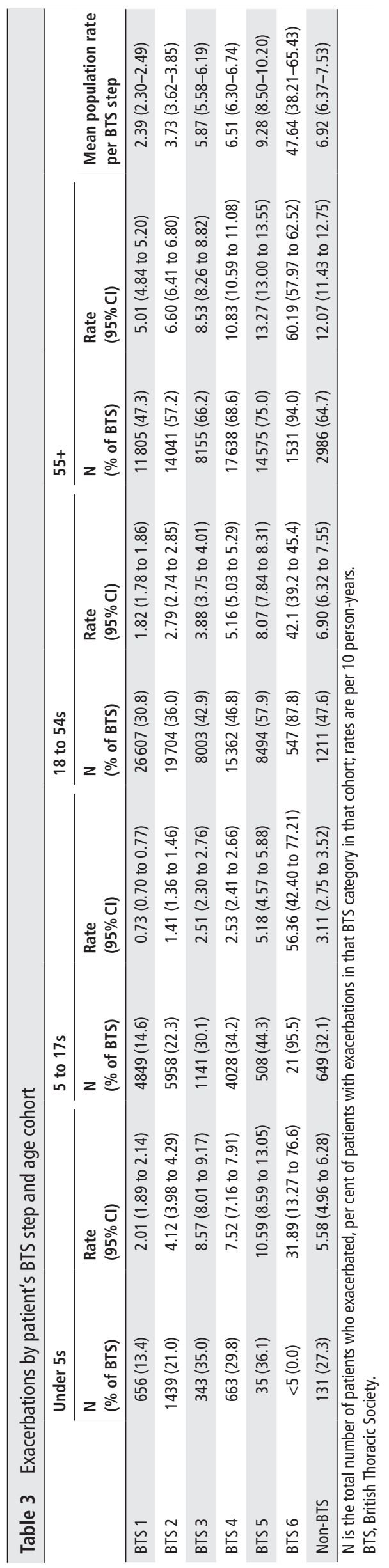

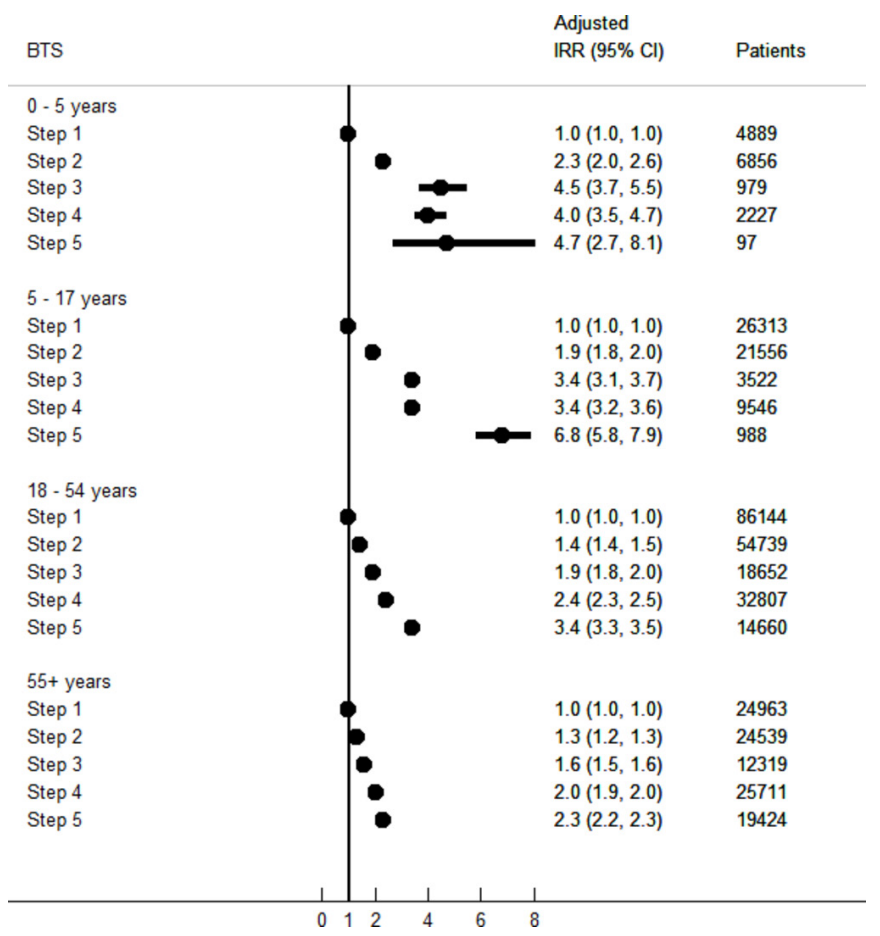

Figure 4 Multivariate regression showing the change in exacerbation rates by BTS step and cohort. All models were adjusted for age, gender, Index of Multiple Deprivation (IMD), atopy, rhinitis, reflux and anxiety; all cohorts except 'Under $5 s^{\prime}$ were also adjusted for smoking and depression (as routinely recorded); ' 18 to $54 \mathrm{~s}$ ' and ' $55+$ ' were also adjusted for body mass index (BMI) and COPD (as missing $\leq 20 \%$ and routinely recorded). Only BTS steps 1, 6 and 5 are shown as these are the main patients with asthma seen in primary care and including BTS step 6 skewed the $x$-axis to an extreme, due to the very wide Cls in two cohorts, such that visual interpretation of the graph was not possible. BTS 6: 'Under $5 s^{\prime}$ IRR $=16.3,95 \% \mathrm{Cl} 0.9$ to 48.0 , patients $<5$; ' 5 to $17 s^{\prime}$ IRR $=59.9,95 \% \mathrm{Cl} 23.7$ to 152.3 , patients 22 ; ' 18 to $54 s^{\prime}$ IRR=16, $95 \% \mathrm{Cl} 14.6$ to 18.3 , patients 635 ; ' $55+$ ' IRR $=10,95 \% \mathrm{Cl} 9.36$ to 10.7 , patients 1629. Rates for BTS step 1 are shown in table 3. BTS, British Thoracic Society; IRR, incidence rate ratio.

asthma in the last 2 or 3 years (depending on their age). However, the level of severity varied considerably between the age cohorts. The youngest and oldest cohorts, $<5$ years old and $\geq 55$ years old, had the lowest proportion on BTS step 1. Only in the 'Under 5s' cohort were patients more likely to have BTS step 2 than 1 ; this may be related to the difficulty in diagnosing asthma in children $<5$ years old (spirometry is often not possible and it can be difficult distinguishing asthma from 'recurrent wheeze') and a reluctance to diagnose until a child is more symptomatic. Asthma was more severe in the ' $55+$ ' cohort, congruent with other older asthma populations. ${ }^{15}$ Nearly a quarter of the ' $55+$ ' cohort had a record indicating concurrent COPD, in keeping with other epidemiology studies ${ }^{16}$; this could be due to misdiagnosis or the presence of features of both diseases (the ' $55+$ ' cohort had a high proportion with a smoking history), nearly two-thirds had their COPD diagnosed after their asthma diagnosis. Currently, there is no published study on the general UK population, but the proportion in each BTS step was similar to a study of a selected sample of UK patients with asthma. ${ }^{17}$

This is the first study to look comprehensively at exacerbation rates in a general asthma population. Rates were highest in the ' $55+$ ' cohort and lowest in the ' 5 to $17 \mathrm{~s}$ ' cohort; in those patients who did exacerbate, the 'Under 5s' cohort had 

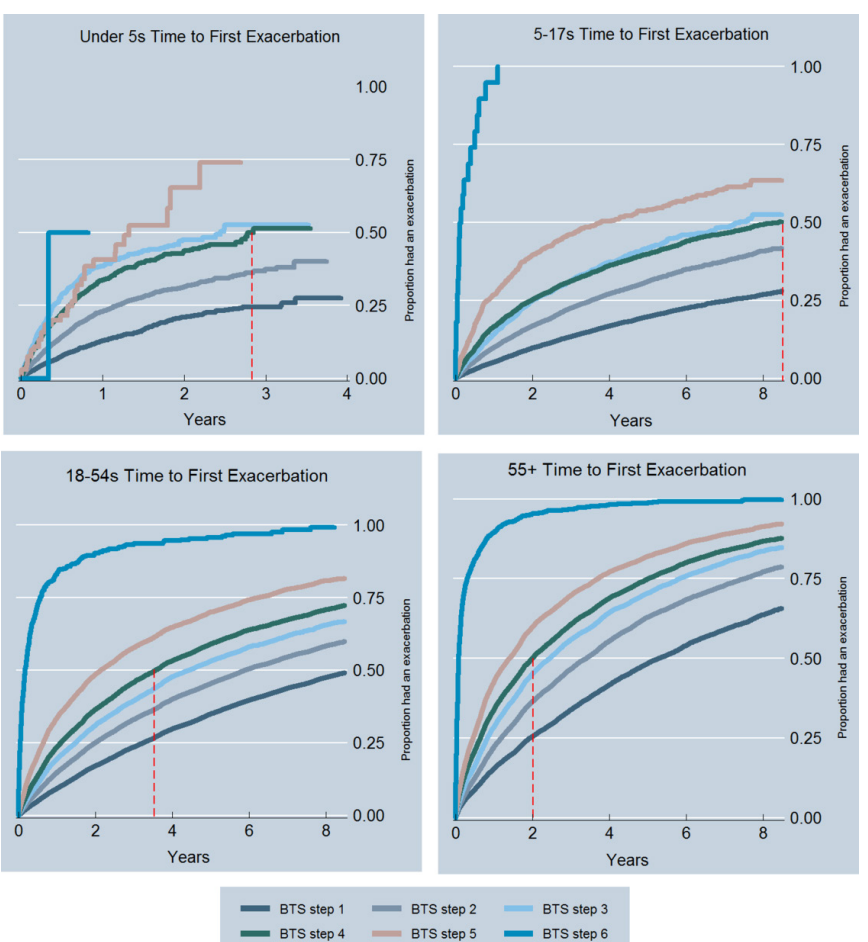

Figure 5 Time to first exacerbation during follow-up by BTS step and cohort. The dotted red line indicates the median time to first exacerbation for patients in BTS step 4. BTS, British Thoracic Society.

the highest proportion of frequent exacerbators. Although the multivariable analysis was adjusted for COPD, there may have been residual confounding in the ' $55+$ ' cohort, including from undiagnosed COPD. For all cohorts, the majority of exacerbations were treated within primary care. The number of asthma deaths was in keeping with reported national statistics. ${ }^{18}$ The rate of Ax-LRTIs in association with an exacerbation was low, supporting BTS guidance not to use antibiotics for an asthma exacerbation, unless clearly clinically indicated. ${ }^{8}$

Many factors significantly changed the rates of an exacerbation, including gender, age, socioeconomic deprivation, smoking history, BMI, atopy history, rhinitis history, reflux history, anxiety history, depression history and COPD history. After adjusting for these factors, increasing BTS step still significantly increased exacerbation rates. The effect was notable in all cohorts, but was more marked, for each incremental BTS step, in those aged $<18$ years compared with those $\geq 18$ years old; suggesting that in childhood asthma the risk of an exacerbation is more influenced by the underlying disease severity than it is in adult asthma.

Cluster analysis has identified a specific phenotype of older patients with asthma with more severe symptoms and worse lung function, ${ }^{19}$ but it is unclear how many older patients with asthma are represented by this phenotype. Our study of a large general asthma cohort (where the age of disease onset is unknown) also suggests a poorer outcome for older patients ( $\geq 55$ years), with both increased disease severity and exacerbation activity. The increase in exacerbations could be explained by the higher proportion with more severe disease, which in turn could be related to the higher proportion with asthma severity risk factors, including a smoking history, coexistent COPD diagnosis and reflux..$^{20-22}$ It has also been postulated to be related to the effects of ageing (including worsening lung function, impaired response to bronchodilators and some changes in immune function), and to longer disease duration in those with early-onset asthma. ${ }^{153}$
Furthermore, the opposite was found for the ' 5 to $17 \mathrm{~s}$ ' cohort which had the least severe asthma and lowest exacerbation activity. There is some evidence to suggest that childhood-onset asthma exhibits a better treatment response and prognosis than adult-onset asthma, ${ }^{24}$ but there is a lack of knowledge regarding a general adult cohort, which would consist of patients with both early and late-onset asthma.

Our findings have shown that there is a strong association between levels of asthma treatment and exacerbation risk. Although this has not been assessed directly elsewhere, it is in keeping with another UK study, and a US study that showed high exacerbation rates in patients despite high-intensity therapy. ${ }^{17}{ }^{25}$ All patients should be on the lowest level of treatment required to achieve their best level of control. Therefore, the question remains whether these findings are due to suboptimal management, poor compliance, poor inhaler technique or other environmental factors, or, if some of these patients have a specific poorly responding phenotype despite maximal pharmacological management.

\section{Limitations}

A possible limitation was misclassification of patients with asthma, but as the Read codes used have a high positive predictive value of $86 \%$ (paper in press), this study may have excluded some patients with asthma but is unlikely to have included many patients who did not have asthma. The study did not include mild exacerbations, for example, treated by the patient themselves, as these are not routinely recorded within CPRD. Using a cut-off of $300 \mathrm{mg}$ for exacerbations may have excluded some exacerbations that were treated for more prolonged periods with high OCS doses; however, from a sensitivity analysis changing the cut-off had a little effect (online supplementary table 6). From 2007 to 2015 , only $62 \%$ of A\&E data were captured by CPRD; this would have reduced the number of A\&E exacerbations in this study, but these were only a small proportion of total exacerbations, so any deficit is unlikely to have significantly affected the findings.

There were some potential limitations in respect to the measurement of BTS step. First, a defined period of 1 year prior to study entry was chosen, but it is possible that some patients' BTS step changed during their follow-up. Second, it is possible some GP practices did not follow BTS guidelines, although most alternative national treatment guidelines (including local Clinical Commissioning Groups guidance, National Institute for Health and Care Excellence guidelines and the British National Formulary) are created using the BTS guideline. It was notable that only a small percentage of patients were not prescribed medication that fitted with the BTS stepwise approach. Lastly, the medications prescribed may not be the medication used, yet it would be expected that the prescription itself indicated the GP's evaluation of the patient's disease severity. The 2016 BTS guidelines are spread over six steps, and were not the ones used by GP practices during the study time period, which predated these guidelines. The 2016 guidelines were selected due to the improved clarity in correlating ICS dosage to asthma severity. Due to the large amount of missing BMI data in the 'Under 5s' and ' 5 to $17 \mathrm{~s}$ ' cohorts, the multivariate regression models for these cohorts did not include BMI.

\section{CONCLUSIONS}

The majority of patients with asthma within the UK have mild disease and infrequent exacerbations, which are managed within primary care. There are distinct differences in characteristics across the generations of patients with asthma, including gender, smoking 
history, atopy and asthma severity. After adjusting for multiple risk factors, the rates of exacerbations are strongly associated with disease severity, as measured using the BTS stepwise approach.

Contributors All authors provided substantial contributions to the work. JKO and $P C$ devised the study. CIB analysed and interpreted the data and produced draft manuscripts. FN provided data input for the analysis. JKQ, PC, LS, FN and ID commented on analysis and manuscript drafts. All were happy with the final version and approved it for submission for publication.

Competing interests None declared.

Ethics approval The protocol for this research was approved by the Independent Scientific Advisory Committee (ISAC) for MHRA Database Research (protocol 16_067); the approved protocol was made available during peer review. Generic ethical approval for observational research using the CPRD with approval from ISAC has been granted by a Health Research Authority Research Ethics Committee (East Midlands-Derby, REC reference number 05/MRE04/87).

Provenance and peer review Not commissioned; externally peer reviewed. (c) Article author(s) (or their employer(s) unless otherwise stated in the text of the article) 2018. All rights reserved. No commercial use is permitted unless otherwise expressly granted.

\section{REFERENCES}

1 Simpson CR, Sheikh A. Trends in the epidemiology of asthma in England: a national study of 333,294 patients. J R Soc Med 2010;103:98-106.

2 To T, Stanojevic S, Moores G, et al. Global asthma prevalence in adults: findings from the cross-sectional world health survey. BMC Public Health 2012;12:204.

3 International Study of Asthma and Allergies in Childhood (ISAAC). The Global Asthma Report 2011. The Global Asthma Report 2014. Int Study Asthma Allerg Child. Int Union against Tuberc Lung Dis 2014.

4 Royal College of Physicians. Why asthma still kills. London: Royal College of Physicians, 2014.

5 Health and Social Care Information Centre. Attribution data Set GP-Registered populations scaled to ONS population estimates, 2012. http://content.digital.nhs.uk/ catalogue/PUB05054

6 Herrett E, Gallagher AM, Bhaskaran K, et al. Data resource profile: Clinical Practice Research Datalink (CPRD). Int J Epidemiol 2015;44:827-36.

7 Nissen F, Quint JK, Morales D, et al. Validation of the recording of asthma diagnosis in UK electronic health records (Clinical Practice Research Datalink). Am J Respir Crit Care Med 2017;195:A2025.
8 British thoracic society. BTS/SIGN British guideline on the management of asthma, 2016. https://www.brit-thoracic.org.uk/standards-of-care/guidelines/btssign-britishguideline-on-the-management-of-asthma/

9 Quint JK, Müllerova H, DiSantostefano RL, et al. Validation of chronic obstructive pulmonary disease recording in the Clinical Practice Research Datalink (CPRD-GOLD). BMJ Open 2014;4:e005540.

10 Anderson HR, Pottier AC, Strachan DP. Asthma from birth to age 23: incidence and relation to prior and concurrent atopic disease. Thorax 1992;47:537-42.

11 Schatz M, Camargo CA. The relationship of sex to asthma prevalence, health care utilization, and medications in a large managed care organization. Ann Allergy Asthma Immunol 2003;91:553-8.

12 Health \& Social Care Information Centre, O. of N. S. Statistics on smoking, 2016.

13 Health Survey England. Adult Weight. Natl Obes Obs 2011.

14 Craig TJ. Aeroallergen sensitization in asthma: prevalence and correlation with severity. Allergy Asthma Proc 2010;31:96-102.

15 Quadrelli SA, Roncoroni A. Features of asthma in the elderly. J Asthma 2001:38:377-89.

16 Postma DS, Rabe KF. The asthma-COPD overlap syndrome. N Eng/ J Med 2015:373:1241-9.

17 Price D, Wilson AM, Chisholm A, et al. Predicting frequent asthma exacerbations using blood eosinophil count and other patient data routinely available in clinical practice. J Asthma Allergy 2016;9:1-12.

18 Office of National Statistics. Asthma deaths in england and wales, 2001 to 2015 occurrences - office for national statistics. 2016 https://www.ons.gov.uk/peoplepopula tionandcommunity/birthsdeathsandmarriages/deaths/adhocs/005955asthmadeaths inenglandandwales2001to2015occurrences.

19 Haldar P, Pavord ID, Shaw DE, et al. Cluster analysis and clinical asthma phenotypes. Am J Respir Crit Care Med 2008;178:218-24.

20 Harding SM. Recent clinical investigations examining the association of asthma and gastroesophageal reflux. Am J Med 2003;115(Suppl 3A):39S.

21 Hardin M, Silverman EK, Barr RG, et al. The clinical features of the overlap between COPD and asthma. Respir Res 2011;12:127.

22 Thomson NC, Chaudhuri R, Livingston E. Asthma and cigarette smoking. Eur Respir J 2004; 24:822-33.

23 Hanania NA, King MJ, Braman SS, et al. Asthma in the elderly: Current understanding and future research needs--a report of a National Institute on Aging (NIA) workshop. $J$ Allergy Clin Immunol 2011;128:S4-S24.

24 de Nijs SB, Venekamp LN, Bel EH. Adult-onset asthma: is it really different? Eur Respir Rev 2013;22:44-52.

25 Schatz M, Meckley LM, Kim M, et al. Asthma exacerbation rates in adults are unchanged over a 5-year period despite high-intensity therapy. J Allergy Clin Immunol Pract 2014;2:570-4. 